\title{
DESAFÍOS EN LA ATENCIÓN SANITARIA DE MIGRANTES INTERNACIONALES EN CHILE
}

\author{
Margarita Bernales ${ }^{1, a}$, Báltica Cabieses ${ }^{1,2, b}$, Ana María Mclntyre ${ }^{1, c}$, Macarena Chepo $^{1, d}$
}

\begin{abstract}
RESUMEN
Objetivos. Indagar en las percepciones de los trabajadores de salud primaria (TSP) sobre los desafíos de atención de salud para población migrante. Materiales y métodos. Se realizó un estudio cualitativo de caso múltiple en ocho comunas de Chile, mediante la técnica de bola de nieve se reclutó a 101 TSP y autoridades locales. Se realizaron entrevistas semiestructuradas y grupos focales, logrando saturación de información. Resultados. Los hallazgos fueron agrupados en dos grandes ejes temáticos: 1. Dificultades técnicas y administrativas, y 2. Percepción de barreras culturales. De acuerdo a los TSP, pese a que se han establecido normativas y se han generado estrategias de atención en salud para población migrante, estas, en su mayoría, no son estables ni conocidas por todos los TSP. Tampoco son fáciles de implementar en las diversas realidades investigadas. La ausencia de registros sobre el número de migrantes que accederán al sistema sanitario, dificulta el diseño de intervenciones específicas. Adicionalmente, la atención en salud presenta complicaciones, y los TSP no necesariamente poseen herramientas que les permitan brindar una atención que sea culturalmente sensible a las necesidades de la comunidad migrante. Conclusiones. Los hallazgos ponen en discusión los nuevos desafíos en salud que enfrenta Chile frente a la creciente población migrante. Las necesidades percibidas por TSP son: estabilidad y claridad en las normativas vigentes en relación a acceso y prestación de servicios, capacitación en normativas y la manera concreta en la que estas deben operar, y sensibilización en competencia cultural.
\end{abstract}

Palabras clave: Migración Humana; Chile; Trabajadores de Salud; Atención Primaria de Salud. (fuente: DeCS BIREME).

\section{CHALLENGES IN THE HEALTH CARE OF INTERNATIONAL MIGRANTS IN CHILE}

\begin{abstract}
Objectives. To investigate the perceptions of primary health workers (PHW) about the challenges of health care for migrants. Materials and methods. A qualitative multicase study was conducted in eight communes of Chile, using the snowball technique, where $101 \mathrm{PHW}$ and local authorities were recruited. Semi-structured interviews and focal groups were conducted, achieving information saturation. Results. The findings were grouped into two major thematic axes: 1. Technical and administrative difficulties, and 2. Perception of cultural barriers. According to the PHW, although regulations have been established and health care strategies have been generated for the migrant population, these are mostly not stable or known to all PHW. They are also not easy to implement in the various realities investigated. The absence of records on the number of migrants accessing the health system makes it difficult to design specific interventions. Additionally, health care has complications, and the PHW do not necessarily have tools that allow them to provide a care that is culturally sensitive to the needs of the migrant community. Conclusions. The findings put into question the new challenges in health that Chile is facing in the face of the growing migrant population. The needs perceived by PHW are: stability and clarity in the regulations in force in relation to access and provision of services, training in regulations and the concrete way in which they should operate, and sensitization in cultural competence.
\end{abstract}

Key words: Human Migration, Chile, Primary Health Care, Health Personnel. (source: MeSH NLM).

\section{INTRODUCCIÓN}

La migración internacional en Chile ha aumentado aceleradamente en los últimos años ${ }^{(1)}$. El Departamento de Extranjería y Migración en Chile, anualmente recopila la información sobre solicitud de visas. Esta información contiene solo los datos de las personas que han ingresado al país de manera regular, por lo que no necesariamente representa el total de los migrantes residentes. Para el período 2014 y 2015, el mayor porcentaje de inmigrantes, a nivel nacional, declaró ser de nacionalidad peruana, el segundo corresponde a

\footnotetext{
Facultad de Medicina Clínica Alemana, Universidad del Desarrollo. Santiago, Chile

Department of Health Sciences, University of York. York, England, UK

a Psicóloga, PhD en Salud Comunitaria; ${ }^{\mathrm{b}}$ enfermera-matrona, PhD Health Sciences (Social Epidemiology); ${ }^{\mathrm{c}}$ tecnólogo médico, MSc Psicología de la Salud; d enfermera, matrona

Recibido: 20/11/2016 Aprobado: 19/04/2017 En línea: 28/06/2017
}

Citar como: Bernales M, Cabieses B, McIntyre AM, Chepo M. Desafíos en la atención sanitaria de migrantes internacionales en Chile. Rev Peru Med Exp Salud Publica. 2017;34(2): 167-75. doi: 10.17843/rpmesp.2017.342.2510 
migrantes colombianos, y en tercer lugar se encuentran los bolivianos. Los siguientes porcentajes son de menor cuantía y están ocupados por migrantes argentinos, haitianos y venezolanos ${ }^{(2)}$. Las restantes comunidades ingresadas al país presentan menos de un $3 \%$ de solicitudes de visa (Figura 1). El 16\% de los inmigrantes que llegan a Chile se establecen en el norte de Chile y el $62 \%$ en la Región Metropolitana ${ }^{(2)}$.

Una proporción significativa de esta población vive actualmente en condiciones de vulnerabilidad social $(3,4)$. Esto ocurre por diversas razones: se encuentran en situación irregular (ingresaron al país en forma ilegal o con visa de turismo, la cual ya se encuentra vencida), pobreza, malas condiciones de vivienda, desempleo o empleo informal y procesos de discriminación y abuso ${ }^{(5)}$. Otra importante razón que sitúa a la población migrante en una posición de vulnerabilidad, es la burocracia que obstaculiza el obtener un documento oficial de identidad de residente en Chile, esencial para acceder al sistema de salud, llamado RUT (Rol Único Tributario).

La vulnerabilidad social de la población migrante regular e irregular en Chile tiene impacto directo en su salud y la de sus familias ${ }^{(6,7)}$, tanto por procesos de migración ${ }^{(8)}$, por condiciones sociales de vida al arribo ${ }^{(9)}$, o por barreras de acceso al sistema de salud formal ${ }^{(10)}$. El acceso a servicios de salud otorgado a la comunidad migrante residente en Chile, en la actualidad, depende -para ciertos servicios- de factores como trabajo, RUT y tipo de visa. El año 2013, un $8,5 \%$ de los inmigrantes del país afirmaban no tener previsión de salud y, en términos de demanda por atención, el $23,7 \%$ de inmigrantes intentó pedir hora por algún problema de salud, pero no la obtuvo, quedando con esta vital necesidad insatisfecha ${ }^{(2)}$.

\section{MENSAJES CLAVE}

Motivación para realizar el estudio. La migración internacional en Chile ha aumentado en los últimos años, en este escenario el sistema de salud y sus trabajadores se ven desafiados a prestar atención a una población muchas veces desconocida para ellos.

Principaleshallazgos. Existen dificultades técnicas y administrativas para la atención sanitaria de migrantes internacionales, pese a que se han establecido normativas y se han generado estrategias de atención en salud, estas en su mayoría no son estables ni conocidas por todos los trabajadores de salud y no necesariamente poseen herramientas que les permitan brindar una atención que sea culturalmente sensible a las necesidades de la comunidad migrante.

Implicancias. Este estudio pretende ser un aporte en la necesaria discusión acerca de los nuevos desafíos en salud que enfrenta Chile frente a la creciente población migrante, siendo necesario entregar atenciones en salud con una comprensión cultural.

Se evidencia un estado de desprotección legal en la salud para parte de la comunidad migrante. En algunos casos existe protección bajo convenios de atención basados en tratados internacionales, por ejemplo, en embarazo y parto. Otra parte de la población presenta vacíos legales para ser atendida a nivel sanitario, algunos quedan al amparo de normativas poco conocidas o que operan como medidas paliativas. Muchas veces la difusión de estas normativas se hace por medio de circulares que no llegan a todos los trabajadores de salud. Un ejemplo cotidiano, relativo a la confusión de información que manejan los trabajadores de la salud, es el desafío de atender a niños extranjeros, que no tienen número de RUT, ya que, si bien ellos tienen derechos legales que aseguran su atención en salud, los sistemas

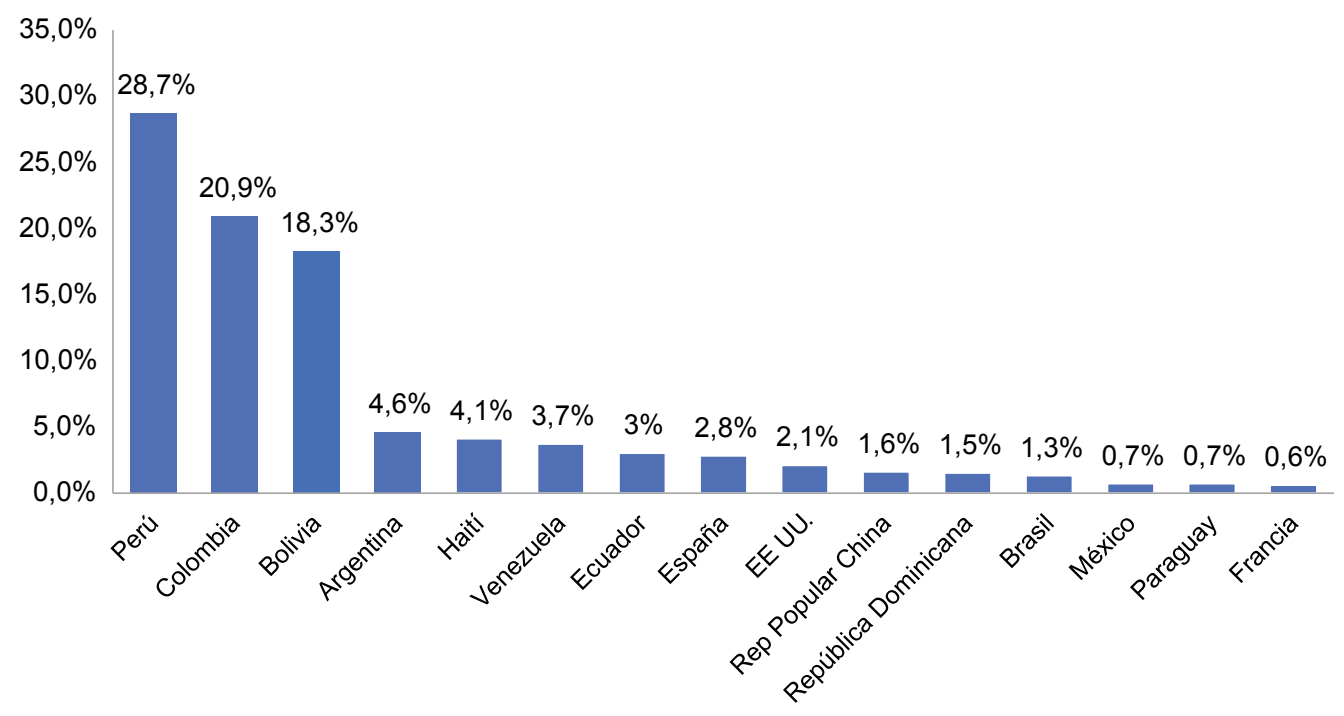

- Visas Otorgadas 2014-2015

Figura 1. Migración reciente por nacionalidades según la cantidad de visas otorgadas 2014-2015 a nivel nacional (Fuente: DEM) (2) 
computacionales de los centros de salud no pueden crear una ficha sin dicho número ${ }^{(2)}$.

Sumado a lo anterior, existen desafíos culturales, que se convierten en barreras para la integración de la población migrante internacional en Chile, representados por diferencias en visiones, tradiciones, estilos de vida, entre otros. Existe amplia evidencia internacional sobre experiencias de estigma y discriminación (11) estrés ${ }^{(12)}$ y problemas de salud mental ${ }^{(13)}$ en población migrante en el mundo. La experiencia migratoria es exigente y compleja en sí misma, pudiendo vulnerar los derechos humanos, el bienestar y la salud de quienes la experimentan ${ }^{(14)}$

En este escenario, el sistema de salud y sus trabajadores se ven desafiados a prestar atención a una población muchas veces desconocida para ellos $(15,16)$. Esta población presenta barreras con la regularización de sus documentos en Chile y el consecuente impacto en el acceso a servicios en general. En la actualidad se reconocen importantes esfuerzos de parte de trabajadores de salud primaria (TSP) para dar soporte al gran número de comunidades inmigrantes que arriban al país y que, al no contar con regularización, muchas veces quedan fuera de los beneficios. Destacan dos iniciativas centrales en esta materia: (i) el Plan Piloto de Acceso a Salud para inmigrantes del Ministerio de Salud en coordinación con diversas entidades públicas, incluyendo el fondo nacional de salud de Chile (FONASA) y atención primaria y secundaria de salud (17), y (ii) la protección legal para atención de salud independiente de la regularidad/irregularidad migratoria, vigente para menores de 18 años, mujeres embarazadas, partos y personas con riesgo vital (Ley de Urgencias).

Los TSP interactúan a diario con una población diversa culturalmente y con dificultades de acceso al sistema de salud chileno. Este estudio tiene como objetivo indagar en las percepciones de los TSP sobre los desafíos de atención de salud a población migrante internacional, con el propósito de entender las necesidades de este grupo y los potenciales ámbitos de acción en que las autoridades sanitarias puedan intervenir.

\section{MATERIALES Y MÉTODOS}

\section{TIPO DE ESTUDIO}

Este estudio cualitativo se sustentó en el paradigma constructivista, el cual establece que el fenómeno de interés o de estudio estaría representado por la construcción e interpretación subjetiva que realizan los diferentes actores en torno a una vivencia, contemplando, incluso en dicha construcción, la perspectiva del equipo de investigación involucrado en el proyecto ${ }^{(18)}$. Este estudio se anidó en el proyecto Fondecyt 11130042 "Desarrollando Inteligencia en Salud Pública para inmigrantes en Chile: un estudio multimétodos" (Conicyt, Gobierno de Chile). Se seleccionó la metodología de estudio de caso ${ }^{(18)}$, la cual está orientada a la búsqueda de la descripción en profundidad de un caso (individuo) o muchas personas que comparten una experiencia, y es esa experiencia la que se convierte en un "estudio de caso". Esta metodología permite dar relevancia a un fenómeno de interés y en torno a dicho fenómeno considerar los puntos de vista de diversos actores sociales. El presente artículo corresponde a un análisis secundario de datos obtenidos en el estudio original, centrado en identificar los desafíos que hoy enfrentan los trabajadores de salud primaria que prestan servicios a la creciente población migrante internacional en Chile.

\section{PROCEDIMIENTOS}

El estudio se desarrolló en ocho comunas con la mayor densidad de población migrante con índices marcados de vulnerabilidad social en Chile (Arica, Iquique, Calama, Antofagasta, Santiago, Recoleta, Independencia y Maipú) ${ }^{(4)}$. Se contempló la realización de entrevistas semiestructuradas (individuales y bipersonales) así como grupos focales a: (i) población migrante, (ii) autoridades comunales y (iii) directores y funcionarios de la salud de centros de atención primaria.

El guión de entrevistas y grupos focales del proyecto original fueron desarrollados en base a dos temáticas principales: ${ }^{(1)}$ la relación entre el sistema de salud público y los migrantes internacionales, y (2) las condiciones de vida en que se encuentra la población migrante en nuestro país, entendiendo que dichas condiciones impactan directamente la salud de esta población. Los guiones de entrevistas fueron adaptados a cada tipo de participante.

La variedad en las técnicas de recolección de datos obedece a la búsqueda de información subjetiva y profunda en relación al fenómeno de estudio, vale decir, la salud y bienestar de la población migrante internacional en Chile, a través de entrevistas individuales y bipersonales, y a la búsqueda de develar interacciones y relaciones establecidas entre los participantes a través de las técnicas de grupo focal. Las entrevistas y grupos focales fueron realizados por el equipo investigador del proyecto original, el cual incluyó enfermeros, psicólogos y antropólogos.

Para el reclutamiento de participantes, se contactaron autoridades de salud de los gobiernos comunales de cada localidad, a través de ellos se gestionó el contacto con los centros de salud primaria seleccionados por ellos 
mismos, de acuerdo a la cantidad de población migrante internacional a la cual entregaban atención, accediendo así a los directores y trabajadores de, al menos, dos centros de salud en cada una de las comunas, los cuales presentaban una mayor densidad de población migrante internacional inscrita -formal o informalmenteen sus dependencias. La recolección de datos se realizó entre el año 2014 y 2016 en dos etapas: levantamiento inicial y confirmación de datos.

\section{PARTICIPANTES}

Se realizaron un total de 40 entrevistas semiestructuradas, 29 de ellas individuales, 2 bipersonales y 8 grupos focales. La Tabla 1 describe a los participantes del estudio. La muestra final fue de 101 participantes: 17 directores de atención primaria, 16 autoridades locales municipales, y 68 TSP. El número final de entrevistas contempló criterios de saturación de información para cada una de las comunas ${ }^{(19)}$, momento definido en el que el material cualitativo recolectado deja de aportar nuevos datos para la comprensión de fenómeno en estudio.

Los criterios de inclusión de la muestra fueron: ser mayor de 18 años, mantener una relación formal de trabajo con el centro de salud o gobierno local, y haber tenido contacto con la población migrante internacional habitante de cada comuna.

Tabla 1. Descripción sociodemográfica de participantes del estudio

\begin{tabular}{|c|c|}
\hline $\begin{array}{l}\text { Total de participantes } \\
\mathrm{n}=101\end{array}$ & $\begin{array}{c}\text { Información } \\
\text { sociodemográfica }\end{array}$ \\
\hline $\begin{array}{l}\text { Directores de centros de } \\
\text { salud primaria }(n=17) \\
15 \text { entrevistas individuales }\end{array}$ & $\begin{array}{l}\text { Sexo: } 11 \text { mujeres, } 4 \text { hombres } \\
\text { Rango de edad: } 27-64 \text {, } \\
\text { promedio: } 47,60 \text { años } \\
\text { Sexo: } 2 \text { mujeres } \\
\text { Rango de edad: } 35-39 \text {, } \\
\text { promedio } 37,50 \text { años }\end{array}$ \\
\hline $\begin{array}{l}\text { Autoridades locales de } \\
\text { salud }(n=16) \\
14 \text { entrevistas individuales }\end{array}$ & $\begin{array}{l}\text { Sexo: } 9 \text { mujeres, } 5 \text { hombres } \\
\text { Rango de edad: } 28-58 \text {, } \\
\text { promedio: } 43,86 \text { años } \\
\text { Sexo: } 2 \text { mujeres } \\
\text { Rango de edad: } 46-47 \text {, } \\
\text { promedio: } 46,50 \text { años }\end{array}$ \\
\hline $\begin{array}{l}\text { Trabajadores de salud } \\
\text { primaria TSP }(\mathrm{n}=68) \\
8 \text { grupos focales }\end{array}$ & $\begin{array}{l}\text { Sexo: } 57 \text { mujeres, } 11 \text { hombres } \\
\text { Rango de edad: } 26-67 \text {, } \\
\text { promedio: } 39,01 \text { años }\end{array}$ \\
\hline
\end{tabular}

\section{ANÁLISIS}

Toda la información proveniente del trabajo de campo se convirtió en material escrito, las entrevistas y grupos focales fueron transcritos palabra por palabra. Este material fue analizado por el equipo de investigación a través de análisis temático ${ }^{(20)}$ utilizando el software NVivo 10. Cada vez que en una entrevista o grupo focal se mencionara la situación de los profesionales de salud primaria y los desafíos que ellos enfrentan en la atención a población migrante internacional, esta se dejó aparte para análisis propio. Esto ocurrió en la mayoría del material cualitativo disponible, aunque fuese muy breve, por lo que se decidió incluir a todos los participantes del estudio. En Tabla 2 se describen las preguntas que fueron seleccionadas para este análisis secundario de información, las categorías que emergieron a partir de dichas preguntas en el proceso de análisis y los códigos emergentes.

\section{CRITERIOS DE RIGUROSIDAD CIENTÍFICA}

Este estudio contempló el cumplimiento de criterios de rigurosidad científica que se han postulado para investigación cualitativa. Estos incluyen: (i) reconocimiento de los propios supuestos del investigador acerca del fenómeno en estudio (delimitados en el proyecto Fondecyt original); (ii) confirmación de hallazgos con una selección de los participantes (que se realizó vía telefónica con un $15 \%$ de la muestra total); (iii) contar con un comité asesor comunitario y un comité asesor académico, que fueron consultados en múltiples oportunidades durante el trascurso del estudio.

\section{CONSIDERACIONES ÉTICAS}

El proyecto original (Fondecyt 11130042) fue revisado y aprobado por el Comité de Ética de la Universidad del Desarrollo y por el Comité de Ética de Fondecyt, Comisión Nacional de Investigación Ciencia y Teconología, Gobierno de Chile.

\section{RESULTADOS}

Los hallazgos de este análisis fueron agrupados en dos grandes ejes temáticos: 1. Dificultades técnicas y administrativas y 2. Percepción de barreras culturales. Entre éstas últimas, la falta de herramientas por parte de los TSP para atender a una población culturalmente diversa y desconocida. Dichas limitaciones van desde el lenguaje hasta las costumbres alimentarias, en cada etapa del ciclo vital, otras, y según lugar de origen del usuario. Diferencias culturales, diversas tradiciones y creencias cruzan todos los ámbitos del quehacer humano, incluyendo la atención de salud en sistemas sanitarios que están organizados de manera diferente de un país a otro, con su propia subcultura 
Tabla 2. Descripción de guía de entrevistas/grupos focales, categorías, códigos emergentes y ejemplo de viñetas de descripción de cada código identificado en el estudio

\begin{tabular}{|c|c|c|c|}
\hline $\begin{array}{l}\text { Guía de entrevistas/ } \\
\text { grupos focales: } \\
\text { ¿qué se preguntó? }\end{array}$ & Categoría & $\begin{array}{c}\text { Códigos } \\
\text { emergentes }\end{array}$ & Viñetas \\
\hline \multirow{2}{*}{$\begin{array}{l}\text { (a) ¿Cómo describirían } \\
\text { la relación entre los } \\
\text { inmigrantes y el centro } \\
\text { de salud o consultorio? } \\
\text { ¿Por qué? }\end{array}$} & $\begin{array}{l}\text { Políticas de } \\
\text { migración }\end{array}$ & $\begin{array}{l}\text { Dificultades en } \\
\text { regularización de } \\
\text { documentos de } \\
\text { residencia }\end{array}$ & $\begin{array}{l}\text { Tú tienes que solamente ir a pararte en la mañana allá a la gobernación y te darás cuenta la cantidad de gente que } \\
\text { hace fila, ahora duermen ahí, para que le den número para tramitar su visa, su RUT, son... mmh millones... hacen } \\
\text { fila, fila fila, fila... Entrevista 53, grupo focal TSP, Calama }\end{array}$ \\
\hline & $\begin{array}{l}\text { Acceso a } \\
\text { centros de } \\
\text { salud }\end{array}$ & $\begin{array}{l}\text { Alto costo de la } \\
\text { vida determina } \\
\text { que migrantes } \\
\text { vivan en malas } \\
\text { condiciones }\end{array}$ & $\begin{array}{l}\text { La alimentación es carísima, luz, el agua, el transporte, es carísimo los arriendos son peores. ¿iTú sabes qué tiene } \\
\text { que hacer la gente!? Se va a campamento (...) en condiciones de inseguridad, recuerda que en los campamentos } \\
\text { se cuelgan a la electricidad, no hay agua potable tampoco hay alcantarillado, todos eso afecta la salud. Entrevista } \\
21 \text {, mujer, TSP, Antofagasta. }\end{array}$ \\
\hline
\end{tabular}

(b) ¿Creen ustedes que los inmigrantes conocen sus derechos en relación a la Chile? migrantes condiciones Confusión

Capacitación del personal frente personal fren a fenomeno migratorio y cultura de los (d) ¿Tienen alguna sugerencia para mejorar el servicio de salud dirigido a inmigrantes en el
Sugerencias para mejorar la salud centro de salud?

\section{(f) ¿Podría}

describirme el proceso

para tomar una

hora en el centro de salud? ¿son procesos diferentes para la población chilena y la inmigrante? ¿De qué forma? ¿Cómo estas diferencias podrían afectar la salud de los inmigrantes?
Políticas de

salud

Políticas de migración

Acceso a centros de salud de atención primaria respecto a políticas públicas

Burocracia y desconocimiento en la implementación de políticas diferente origen

Conocer datos demográficos y detectar y detectar
necesidades necesidades eventualmente
Que no sean atenciones fantasmas, que queden como prestaciones y quede el respaldo legal de cada una de ellas, y no existe la forma. Y el Ministerio, si bien es cierto nos dice: hay que atenderlo... Pero no nos da la logística. Entrevista 53, grupo focal TSP Calama

..largas filas de de... inmigrante. Eeeh ahí corre muchas veces el criterio personal, profesional, criterio deee, en este caso, por ejemplo, si el centro de salud pertenece a la municipalidad, cuáles son las políticas que va a implementar respecto a los inmigrantes. Entrevista 8, hombre, TSP, Maipú.

El otro desafío que tenemos es importante, es un poco capacitar al funcionario en todos estos modelos transculturales, como poder entender a este extranjero que viene con unas ideas diferentes, que viene con raíces distintas, aceptar también. ... O sea, empezar a capacitar a los funcionarios en relación a eso, para también, como ya es una población nuestra, ya es nuestra, para poder trabajar con ellos y poder aumentar el grado de satisfacción, o, la percepción de satisfacción de esos pacientes. Entrevista 53, grupo focal de TSP, Calama. La radiografia de cadera para detectar una displasia de cadera en una guagua pequeña mucho no se la hacen, no se la toma, entonces también nosotros ¿Cómo? ¿qué pasa aquí porque ellos se preocupan tanto de ir al dentista que la mamografía, pero la guagua de dos meses no la llevan a la radiografía de cadera y resulta que la guagua tiene 4 meses y tiene displasia y ya tienen que ponerle correa porque no fue a tiempo. Entrevista 12, grupo focal trabajadores de salud, Santiago.

Los desafíos que se vienen nosotros lo vemos por la parte de tratar de aprender más de su de su cultura y de sus Los desafios que se vienen nosotros lo vemos por la parte de tratar de aprender más de su de su cultura y de sus
convivencias y tratar de quizás ponerle algo especial es esa atención (l: yaa) en ese sentido... Tan sencillo cómo vamos a preparar la papilla del bebe (l: $\mathrm{mmm}$ claro es súper importante...) Nosotro... nosotros le vamos a dar puré de manzana, sin embargo, pareciera que ellos lo primero que le dan es una papa por ejemplo (l: claro...) entonces ahi (I: que haya congruencia entre el discurso de salud yy..) Claro si le damo, si le damo un discurso de al... de primera alimentación tan estructurado como lo tenemos acostumbrado nosotros que es lo que hacemo hasta ahora y lo más probable es que poco poco no van escuchar o poco lo van hacer lo que le decimo y van a llegar a hacerle y lo más probable es que poco poco no van escuchar o poco lo van hacer lo que le decimo

Pasa con los colombianos. Como uno recién está conociendo la forma que ellos tienen, en cambio con los peruanos $P a s a$ con los colombianos. Como uno recién está conociendo la forma que ellos tienen, en cambio con los peruanos
estamos acostumbrados. Pero con los colombianos no sabemos si están enojados, vienen de forma agresiva me está hablando o si la persona es asi. Por ejemplo, con los peruanos sabemos que tutean a todos, ellos no tratan a nadie de usted, entonces es como ya el hábito de saber cómo ellos son, pero con los colombianos, son agresivos. Él habla fueeerte, porque llega y les haabla, digamos exigiendo su atención. Entonces eso como que produce una choque con el equipo de salud [l: $\mathrm{mm}$, no están acostumbrados a enfrentar, o sea para nosotros ya es alguien agresivo son los hombres que levantan la voz. Faltan estudios todavia para analizar la población. ... Choque intercultural entre los trabajadores de salud y los migrantes, que ellos saben cuáles son sus derechos, los conocen. Saben que los tenemos que atender. ... Son muy exigentes, es sorprendente... En cambio, los chilenos estamos recién aprendiendo del tema. Entrevista 67 , mujeres, autoridades de salud, lquique.

O muchas veces no dan credibilidad a los diagnósticos que tú les estás dando, a mi me pasa con las personas, por ejemplo, las bolivianas, cuando tú las notificas de Chagas: No, yo no tengo Chagas... iNo! pero sí, tienen, y vienen y te dicen que ino!.. No si yo no tengo Chagas, me voy a tomar unas hierbitas y voy no sé, al santito... así tienen todo un tema y pierdes adherencia a esa persona, entonces si tú eres demasiado, logras poner una barrera, y te dicen: un tema y pierdes adherencia a esa persona, entonces si tú eres demasiado, logras poner una barrera, y te dicen:
No, eso no... y te vas así, el paciente no vuelve más y es asi de simple. Entrevista 53, grupo focal trabajadores de No, eso no... y
salud Calama

salud Calama hablamos muy rápido. Y a veces no entienden las indicaciones. Ahora obviamente, si yo fuera... hay modismos que tenemos nosotros que tampoco entienden, entonces a veces la comunicación es la que lleva a errores, la presentación de los medicamentos, a veces en otro país es diferente a la nuestra.... Ahora, depende de la gente que venga acá, depende del pais que sean. Generalmente, los usuarios ecuatorianos o colombianos si son, entienden un poco más los temas, a veces nos pasa... y yo también en la sala (no se entiende) atendí a niñitos. Y muchas veces me costaba mucho, por ejemplo, que una mamá me entendiera cómo usar el inhalador con la Y muchas veces me costaba mucho, por ejemplo, que una mama me entendiera cómo usar el inhalador con la de urgencia. Eehh... la comunicación a veces nos falla. Entrevista 22, hombre, director de CESFAM, Antofagasta. Poder capacitar a los funcionarios de los centros de salud con relación a los migrantes, conocer cuáles son ... tener un diagnóstico acabado de cuál es la realidad con relación a los migrantes en la comuna, porque sabemos que más o menos son como 120, sabemos, pero no hay un estudio o una cifra concreta desde la municipalidad que nos diga tantos migrantes son, tantos son peruanos, son estudios que se han hecho, pero son todos con resultados más bien tantos migrantes son, tantos son peruanos, son estudios que se han hecho, pero son todos con resultados más bien la población migrante, Entrevista 99, grupo focal de trabajadores de salud, Independencia.

También está este tema: de que las podamos ingresar sin el RUT, significa que no le pagan per cápita a salud... por lo tanto, sin embargo, se les está entregando igual una atención y eso... se supone que se paga por atención... pero como no tienen RUT, no se pueden ingresar las acciones... y dejan de ingresar recursos.... Entrevista 99, grupo focal TSP, Independencia.

Entonces se tiene una meta que nosotros no vamos a cumplir. Y que no cumplimos nunca por lo mismo. Entonces, el tema de las dentales... igual. No pueden ser ingresadas como al SIGES y al sistema, porque no son GES ... Porque

Dificultades la ley del GES es superclara, quién tiene derecho al GES es el usuario que tiene Fonasa, y tiene un RUT chileno relacionadas a la Claro, pero después igual te llegan los oficios y tienes que darle todas las prestaciones... Claro, y son prestaciones

cápignación per del centro al menos a nosotros como funcionarios, es un tema que siempre se presta para hacer catarsis ... iPero al final los cápita del centro de salud atendemos igual! Entrevista 53, grupo focal trabajadores de salud, Calama.

Todo lo que es inmigrante, recién estamos en eso. Hemos tenido reuniones con el servicio, con el SEREMI, con el encargado de no sé qué, con la encargada que viene... y siempre llegamos a lo mismo, que hay que ver y todo queda en lo mismo, vamos a ver, vamos a mandar, vamos a enviar, y ahí quedamos siempre, entonces en esos protocolos como internos que hacemos después vienen ellos: eso no se hace, después hacemos otro: no se hace, después viene otra tipa más y nos dicen la misma cháchara. Entrevista 48, mujer, autoridad de salud, Calama. 
y normativas. A continuación, se expone la síntesis de los desafíos encontrados, agrupados en las dos categorías principales (ver Tabla 2 para complementar los hallazgos).

\section{DIFICULTADES TÉCNICAS Y ADMINISTRATIVAS}

Respecto del desconocimiento de cuántos y quiénes son los migrantes potenciales del sistema de salud, los TSP mencionan que la ausencia de cifras fidedignas conlleva una larga cadena de dificultades para la atención. Estas van desde tareas básicas como la planificación y destino de recursos a nivel comunal, pasando por los montos que deben asignarse a ciertos programas de salud y terminando en la ausencia de importantes datos demográficos de la población atendida.

Yo creo que el primer desafío es conocer la real población que tenemos, para poder destinar los recursos, pero... no tener una población de la cual podemos tomarnos, es difícil programar, destinar recursos. Entrevista 42, mujer, directora del de Centro de Atención Primaria de Salud Arica

Otro desafío relevante en el discurso de los TSP es la falta de recursos económicos para atender a la población. En la opinión de TSP, la falta de recursos se vincula a que muchos migrantes no están inscritos en el sistema de salud, dado que no tienen regularizada su situación migratoria; en Chile, el cálculo de la asignación de recursos a los centros de salud se da de acuerdo al número de RUT de sujetos atendidos durante un periodo determinado. En el caso de la población migrante sin RUT, no existe una asignación de recursos estatales per cápita, lo que sobrecarga al sistema y no permite incorporarlos en los registros electrónicos.

Ha sido un costo que hemos tenido que asumir con las mismas platas fiscales y sin recibir ningún aporte por ello (...). Ahora, obviamente, hay algunos extranjeros que están regularizados..., pero hay un gran número de personas que no cumplen con eso y que hay que otorgarle, de igual manera, todas las prestaciones que estén incorporadas en la atención a esos grupos vulnerables... o definidos como grupos de riesgo. Entrevista 48, mujer, autoridad de salud, Calama.

Con frecuencia los TSP entrevistados expresan su frustración ante normativas dictadas de manera centralizada. Estas son percibidas como normativas poco claras y difíciles de implementar.

Mira, yo creo que ha generado, de alguna manera, en el equipo de salud, igual un poco de decepción, porque igual ellos [TSP] ven cómo ellos [migrantes] acceden a través de acuerdos internacionales que los hacen los presidentes y la gente que está como a millones de escalones de nosotros [TSP] y que a la larga tú ves que va de alguna manera en desmedro de tu bien o de tus recursos. Entrevista 48, mujer, autoridad de salud, Calama

A nivel de atención primaria de salud, los TSP se han ido adaptando a la demanda de atención de la población migrante. Han ideado mecanismos para ingresar a los pacientes y así cumplir las normativas internacionales exigidas a nivel central. A nivel local (comunal) y de manera coordinada entre autoridades municipales y centros de salud, se intenta regular la atención de migrantes y mejorar su calidad y cobertura. Por ejemplo, en algunas comunas se instruye al personal para que todo migrante pase, inicialmente, por una atención de ingreso al sistema de salud; le entregue información sobre sus deberes y derechos, indague sus condiciones de vida, entre otros. Esta atención inicial se ha implementado por medio de asistentes sociales. Sin embargo, para atención hospitalaria (nivel secundario) las barreras son aún mayores.

[EI RUT] es un tremendo problema, nosotros por, no podemos subir pacientes, por ejemplo, pacientes que son inmigrantes que vienen con diabetes, con hipertensión... Puedo inscribir [en centro de salud primario] un paciente con, con eeh... pasaporte, pero llegan al nivel secundario y al no tener RUT, no los atienden, no sé si los atienden o no los atienden, yo creo que no los atienden. Entrevista 101, mujer, directora del Centro de Atención Primaria de Salud, Recoleta.

La percepción generalizada entre los TSP es que falta información a diferentes niveles. Por una parte, datos estadísticos de cuántos y quiénes son los migrantes beneficiarios de los centros de salud. En segundo lugar, en ámbito de políticas públicas, falta claridad y estabilidad en normas de atención de salud del país y cómo adecuarlas a la realidad del momento. Por otra parte, los TSP perciben que algunos migrantes carecen de información sobre el sistema de salud chileno y los beneficios que puede brindar. Esta falta de información genera expectativas desmedidas, dificultades de atención, pérdida de recursos, deterioro de las relaciones interpersonales, falencias en el acceso a salud y, finalmente, en la salud. Cabe destacar que las percepciones respecto a las dificultades técnicas y administrativas que impactan en el acceso y atención de la población migrante, son compartidas por las autoridades locales y los trabajadores de la salud de todas las comunas incluidas en el estudio.

\section{PERCEPCIÓN DE BARRERAS CULTURALES}

De acuerdo con los participantes de este estudio, para lograr una buena relación entre proveedor de salud y usuario, además de solucionar los temas 
administrativos y técnicos señalados previamente, es fundamental comprender la cultura, costumbres y lenguaje del paciente que se está atendiendo. Los TSP, con frecuencia, enfrentan un mundo culturalmente ajeno y desconocido; con esfuerzo han ido adaptándose en base a sus propias experiencias y creatividad. Ejemplos de ello son las creencias sobre nutrición por parte de la población migrante, o barreras de lenguaje que dificultan la comunicación.

Las peruanas y las bolivianas les dan [al recién nacido una mezcla de harina, cereales, con agua incluso algunas con leche y eso se lo dan como una vez al día (...) desde el mes. Entonces hay niños que parten al tiro con desnutrición o un riesgo, porque realmente le crea un problema de insuficiencia intestinal. Entrevista 25, grupo focal de TSP, Antofagasta.

Usamos mucho modismo al expresarnos, tenemos mucho lenguaje propio..., uno explica cosas como más técnicas, más de, no sé, de qué le va a pasar, que va a tener fiebre, va a tener no sé, alguna reacción y ellos no entienden lo que yo les estoy diciendo, entonces... a uno que atiende como bastante gente... yo no me puedo tomar todo el tiempo del mundo para explicarle a una persona 3, 4, 5 veces lo mismo. Entrevista 12, grupo focal TSP, Santiago.

También llama la atención de los TSP, el ingreso tardío a control prenatal de las mujeres migrantes, atribuyendo dicho retraso a creencias culturales propias de cada grupo.

Controlan su embarazo de forma tardía, nunca llegan antes de las 14 semanas a pesar de que estas mismas peruanas, colombianas... pacientes extranjeras, algunas que van a tener su segundo bebe y uno les enseña que deben venir antes a sus controles. Entrevista 25, grupo focal de TSP, Antofagasta.

TSP y directores de centros de salud tienen bastante claras necesidades y eventuales estrategias de solución a nivel central. Emerge con fuerza la necesidad de sensibilización de los TSP, compartida en todas las comunas que fueron parte de este estudio, acerca del fenómeno migratorio y todo lo que conlleva y las carencias en competencia cultural. Estas carencias afectan directamente la atención clínica con el usuario, por lo cual son reportadas principalmente por los TSP y no de la misma forma por las autoridades locales, sin embargo, durante el proceso de confirmación de resultados, al contrastar esta información con todos los grupos, las autoridades locales de todas las comunas logran visualizar esta problemática y comprenden la importancia de generar estrategias culturalmente pertinentes para la población migrante.
En resumen, los TSP se ven enfrentados a constantes desafíos. Algunos de estos pueden ser abordados por medio del aprendizaje, experiencia e interés personal de mejorar la calidad de la atención. Otros desafíos igualmente importantes, no dependen de la voluntad o conocimiento de los TSP, como la obtención del número RUT o el poder ingresar de manera regular a determinados programas de atención de salud. Las dificultades que se presentan para regularizar la situación migratoria dentro del país son reportadas de manera frecuente como una barrera que obstaculiza el inicio de cualquier solución en cuanto al acceso al sistema de salud formal.

\section{DISCUSIÓN}

De acuerdo a los TSP, el creciente aumento de la población migrante es actualmente un desafío para todo el sistema de salud. La percepción general es que, pese a que se han establecido normativas y se han generado estrategias de atención en salud, estas, en su mayoría, no son estables, ni conocidas por todos. Hay importantes dificultades para implementar normativas de atención de salud en las realidades y culturas locales. Sumado a lo anterior, la ausencia de registros claros en términos del número de migrantes que accederán al sistema de salud dificulta aun más el diseño de intervenciones específicas. Más allá de los problemas de acceso, la atención en sí misma presenta complicaciones, ya que los TSP no necesariamente poseen las herramientas que les permitan brindar una atención en salud que sea culturalmente sensible a las necesidades de la comunidad inmigrante.

Cualquier sistema de salud es alterado en sus funciones frente a la llegada de nuevas comunidades, viéndose desafiado a implementar nuevas estrategias, Estas idealmente deben darse en un marco de competencia cultural por parte de los TSP ${ }^{(21,22)}$. Actualmente en Chile, los aspectos legales y administrativos están siendo abordados y son una preocupación del gobierno central ${ }^{(17)}$. Sin embargo, la capacitación en estrategias que permitan entregar atención en salud culturalmente sensible es escasa.

En la revisión de literatura internacional, se presentan diferentes estrategias de adaptación cultural dirigidas específicamente a la población migrante internacional, y cuyo foco es lograr sistemas de salud sensibles a las diferencias culturales ${ }^{(23-26)}$, a la luz de estos estudios, es posible establecer que las percepciones de los TSP en Chile respecto a la necesidad de recibir formación específica respecto a cómo elaborar estrategias sensibles a la diferencia cultural con diversas comunidades pareciera ser acertada, en tanto la pertinencia cultural de las estrategias en salud pareciera ser una ventaja en la relación que establece cualquier sistema de salud con comunidades migrantes internacionales. 
Las necesidades concretas y urgentes que surgen de este estudio por parte de TSP son las siguientes: (i) estabilidad y claridad en las normativas vigentes en relación a acceso y prestación de servicios; (ii) capacitación en normativas vigentes y la manera concreta en la que estas deben operar, y (iii) sensibilización en competencia cultural. Países con más años de experiencia en la recepción de comunidades migrantes internacionales, tales como Canadá, han reflexionado con mayor profundidad acerca de cómo sus propios sistemas de salud están o no preparados para la recepción y atención oportuna de comunidades migrantes internacionales ${ }^{(27,28)}$. Chile se enfrenta hoy al gran desafío de repensar un sistema de salud que sea claro, inclusivo y pertinente para las nuevas comunidades que están llegando. Tanto los sistemas de salud como sus trabajadores se enfrentan hoy a nuevas comunidades, con necesidades en salud que pueden ser, a su vez, muy similares o muy diferentes a la población local, experimentadas y vivenciadas desde lo subjetivo que cada cultura aporta a los individuos, por ende, en la creación, diseño y reflexión de nuevas estrategias debieran estar presentes las autoridades de nivel central y local, así como también los representantes de las comunidades de migrantes internacionales presentes en Chile.

La limitación que presenta este estudio es no tener la capacidad de captar la evolución del proceso dinámico; por una parte, de la adaptación intercultural de los TSP y, por otra parte, de la compleja implementación de normativas de atención de migrantes en atención primaria. Ambos procesos evolucionan día tras día, pudiendo presentar mayores dificultades o idealmente mejorar.
En conclusión, este estudio pretende ser un aporte en la necesaria discusión acerca de los nuevos desafíos en salud que enfrenta Chile frente a la creciente población migrante. Los hallazgos sitúan a los TSP en una posición de vulnerabilidad, en que se ven exigidos desde distintos flancos: (i) desde las autoridades sanitarias: a sumar a sus tareas diarias estrategias de salud para la población de migrantes internacionales, utilizando los mismos recursos económicos con los que contaban en forma previa a esta nueva demanda; (ii) desde nivel central: se les exige que implementen normativas sin haber tenido participación en su redacción, sin recibir capacitación sobre los procedimientos a seguir, o sobre cómo poner en práctica en concreto tales acciones dentro de un centro de salud, y (iii) desde la población migrante: a entregar atenciones en salud para las cuales se requiere de adaptación cultural, sin haber recibido formación o capacitación para su éxito.

Agradecimientos: a los TSP de las ocho comunas seleccionadas, quienes nos mostraron las dificultades $y$ desafíos que perciben a diario al atender a la comunidad migrante y a los directores de centros de atención primaria de salud que deben dar respuesta a los TSP, a los migrantes y simultáneamente dar cumplimiento a metas sanitarias y normativas internacionales.

Fuente de financiamiento: Proyecto Fondecyt 11130042 "Desarrollando Inteligencia en Salud Pública para inmigrantes en Chile: un estudio multimétodos".

Conflictos de interés. los autores declaran no tener conflictos de interés.

\section{REFERENCIAS BIBLIOGRÁFICAS}

1. Departamento de Extranjería y Migración. Ministerio del Interior y Seguridad Pública. Visas solicitadas y otorgadas 2015. Available from: http://www.extranjeria.gob.cl/.

2. Cabieses B, Bernales M, McIntyre A, editors. La migración internacional como determinante social de la salud en Chile: evidencia y propuestas para políticas públicas. Santiago, Chile2017. Available from: http://www.udd.cl/

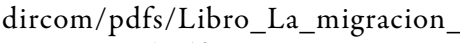
internacional.pdf

3. Cabieses B, Tunstall H, Pickett K. Understanding the socioeconomic status of international immigrants in Chile through hierarchical cluster analysis: a population-based study. International Migration.
2015;53(2):303-20. Available from: http://onlinelibrary.wiley.com/ doi/10.1111/imig.12077/full

4. Vasquez A, Cabieses B. Where are vulnerable immigrants located in Chile? A spatial analysis of Census data using an Index of Multiple Deprivation from the last three decades (1992-2012). Plos One. 2015:under review. Available from: http://journals.plos.org/plosone/ article?id=10.1371/journal.pone.0146047

5. Cabieses B, Tunstall H. Immigrant health workers in Chile: is there a Latin American "brain drain"? Rev Panam Salud Publica. 2012;32(2):161-7. Available from: http://www.scielosp. org/scielo.php?script $=$ sci_arttext\&pi$\mathrm{d}=$ S1020-49892012000800012

6. Cabieses B, Pickett KE, Tunstall H. Comparing sociodemographic factors associated with disability between immigrants and the Chileanborn: are there different stories to tell? International journal of environmental research and public health. 2012;9(12):4403-32. Available from: http://www.mdpi.com/16604601/9/12/4403

7. Cabieses B, Tunstall H, Pickett $\mathrm{K}$. Testing the Latino paradox in Latin America: a population-based study of Intra-regional immigrants in Chile. Revista medica de Chile. 2013;141(10):1255-65. Available from: http://www.scielo.cl/pdf/rmc/ v141n10/art04.pdf

8. Agar L. Migraciones, Salud y Globalización: entrelazando miradas Chile: Ministerio de Salud / Organización 
Internacional para las Migraciones / Organización Panamericana de la Salud. Santiago, 2010. Available from: file://C:/Users/Investigador/Downloads/Migraciones, + Salud + y+Globalizaci\%C3\%B3n+2010.pdf

9. Cabieses B, Bustos P. Vulnerabilidad social en salud por parte de migrantes internacionales en Chile. In: Cabieses Bea, editor. Vulnerabilidad social y sus efectos en salud en Chile. Section III, chapter IV. Santiago: UDD; 2016. Available from: http:// medicina.udd.cl/files/2016/03/LibroCompleto-2016.pdf

10. Cabieses B, Tunstall H, Pickett KE, Gideon J. Understanding differences in access and use of healthcare between international immigrants to Chile and the Chilean-born: a repeated crosssectional population-based study in Chile. Int J Equity Health. 2012;11:68. Available from: https://www.ncbi. nlm.nih.gov/pubmed/23158113

11. Morawa E, Erim Y. [The interrelation between perceived discrimination, depressiveness, and health related quality of life in immigrants of Turkish and Polish origin]. Psychiatrische Praxis. 2014; 41(4):200-7. Available from: https://www.ncbi.nlm.nih.gov/ pubmed/23868716

12. McClure HH, Snodgrass JJ, Martinez CR, Jr., Eddy JM, Jimenez RA, Isiordia LE. Discrimination, psychosocial stress, and health among Latin American immigrants in Oregon. American journal of human biology : the official journal of the Human Biology Council. 2010;22(3):421-3. Available from: https://www.ncbi. nlm.nih.gov/pubmed/19844904

13. de Vries WM, Smits CH. [Searching for rest in one's soul: the experience of mental health complaints in older Moroccan immigrants]. Tijdschrift voor gerontologie en geriatrie. 2005;36(5):194-202. Available from: https://www.ncbi.nlm.nih.gov/ pubmed/16350528

14. Martin SS. Exploring discrimination in american health care system: perceptions/experiences of older Iranian immigrants. Journal of crosscultural gerontology. 2012;27(3):291-
304. Available from: https://www. ncbi.nlm.nih.gov/pubmed/22735985

15. Kleinman A. Local Worlds of Suffering: An Interpersonal Focus for Ethnographies of Illness Experience. Qualitative Health Research. 1992;2(2):127-34. Available from: http://journals.sagepub.com/doi/ abs/10.1177/104973239200200202

16. Kleinman A, Eisenberg L, Good B. Culture, illness, and care: clinical lessons from anthropologic and crosscultural research. Annals of Internal Medicine [Internet]. 1978; 88(2): [2518 pp.]. Available from: http://www. ncbi.nlm.nih.gov/pubmed/626456.

17. MINSAL;FONASA; Serviciode Salud. Orientaciones Técnicas: Programa Piloto de Salud de Inmigrantes. In: Chile Gd, editor. Santiago: MINSAL; 2015. Available from: http://www. minsal.cl/salud-del-inmigrante/

18. Creswell J. Philosophical, Paradigm, and Interpretative Frameworks. In: Creswell J, editor. Qualitative Inquiry and Research Design: Choosing Among Five Approaches. Thousand Oaks: Sage; 2007. p. 15-34. Available from: https://us.sagepub.com/en-us/ nam/qualitative-inquiry-and-researchdesign/book235677

19. Patton M. Variety in Qualitative Inquiry: Theoretical Orientations. Qualitative Research \& Evaluation Methods 3ed: Thousand Oaks; 2002. Available from: https://us.sagepub. com/en-us/nam/qualitative-researchevaluation-methods/book 232962

20. Thomas D. A general inductive approach for qualitative data analysis2003 April 4, 2011:[1-11 pp.]. Available from: http://www.fmhs. auckland.ac.nz/soph/centres/hrmas/_ docs/Inductive2003.pdf.

21. Bernales M, Pedrero V, Obach A, Perez C. [Cultural Competence in health: an urgent need for health workers]. Revista medica de Chile. 2015;143(3):4012. Available from: http://www. scielo.cl/scielo.php ? pid $=$ S003498872015000300018\&script=sci_arttext

22. Restrepo O. Inmigración y etnosalud: Posibilidad de un modelo diferencial de salud. Revista Facultad Nacional de Salud
Pública. 2009;27(1). Available from: http:// www.redalyc.org/ articulo.oa?id=12027103

23. Lindert J, Schouler-Ocak M, Heinz A, Priebe S. Mental health, health care utilisation of migrants in Europe. European Psychiatry. 2008;23:14-20. Available from: https://www.ncbi. nlm.nih.gov/pubmed/18371575

24. Beach MC, Price EG, Gary TL, Robinson KA, Gozu A, Palacio A, et al. Cultural Competency: A Systematic Review of Health Care Provider Educational Interventions. Medical care. 2005;43(4):356-73. Available from: https://www.ncbi.nlm.nih.gov/ pmc/articles/PMC3137284/

25. Hultsjö S, Hjelm K. Immigrants in emergency care: Swedish health care staff's experiences. International Nursing Review. 2005;52(4):276-85. Available from: https://www.ncbi. nlm.nih.gov/pubmed/16238724

26. Castro A, Ruiz E. The effects of nurse practitioner cultural competence on Latina patient satisfaction. Journal of the American Academy of Nurse Practitioners. 2009;21(5):278-86. Available from: https://www.ncbi. nlm.nih.gov/pubmed/19432912

27. Swinkels H, PottieK, Tugwell P, RashidM, Narasiah L. Development of guidelines for recently arrived immigrants and refugees to Canada: Delphi consensus on selecting preventable and treatable conditions. Canadian Medical Association Journal. 2011;183(12):E928-E32. Available from: https://www.ncbi.nlm.nih.gov/ pubmed/20547714

28. Tiagi R. Access to and utilization of health care services among Canada’s immigrants. International Journal of Migration, Health and Social Care. 2016;12(2):14656. Available from: http://www. emeraldinsight.com/doi/abs/10.1108/ IJMHSC-06-2014-0027

Correspondencia: Margarita Bernales

Dirección: Facultad de Medicina Clínica Alemana, Universidad del Desarrollo, Santiago, Chile. Avenida La Plaza 680 San Carlos de Apoquindo Las Condes, Santiago, Chile.

Correo electrónico:margaritabernales@udd.cl 